\title{
Review
}

Mengjiao $\mathrm{Hu}^{1}$, Zhenfei Wang ${ }^{2 \star}$

\section{Treatment of Helicobacter pylori-associated gastritis using traditional Chinese medicine}

DOI: $10.2478 / \mathrm{ii}-2018-0009$

Received January 28, 2018; accepted February 06, 2018; published online May 15, 2018

\begin{abstract}
Helicobacter pylori is a pathogenic bacterium with strong pathogenicity, which can induce chronic gastritis and peptic ulcer in addition to causing gastric mucosal atrophy and intestinal metaplasia after longterm infection. It is even associated closely with the occurrence of gastric cancer and mucosa-associated lymphoid tissue lymphoma. Recently, the incidence of $H$. pylori-associated gastritis has increased rapidly worldwide, especially in China. The prevention and treatment of $H$. pylori infection has become an urgent issue to be resolved by the medical community. Traditional Chinese medicine and the combination of traditional Chinese medicine and Western medicine have recently shown unique advantages in the treatment of $H$. pylori-associated gastritis. The progress in this area is summarized in this review.
\end{abstract}

Keywords: Helicobacter pylori, gastritis, Chinese medicine, combination of traditional Chinese medicine and Western medicine

\section{Helicobacter pylori}

The Gram-negative bacterium Helicobacter pylori was first discovered in 1983 by Barry Marshall and Robin Warren. It is a spiral-shaped microorganism [1-3] living in the stomach and duodenum, and it requires highquality conditions for growth. It can cause either chronic nonatrophic gastritis in the stomach mucosa [4] or gastric and duodenal ulcers; it may even be related to the occurrence of gastric cancer [5-7].

\section{H. pylori-associated gastritis}

Recently, H. pylori infection has been closely associated with chronic gastritis and gastroduodenal ulcers [8]. Modern medicine suggests that [9] the main causes of chronic gastritis and gastroduodenal ulcer are the clinical diseases caused by increased aggressive factors or weakened protective factors. Even more, the imbalance between aggressive factors and protective factors aggravates the disease. China has a high and increasing incidence of chronic gastritis, and 39\%-83\% of the chronic gastritis cases are caused by $\mathrm{H}$. pylori infection [10,11]. Clinically, combinations of three or four antibiotics are usually used in Western medicine to treat $H$. pylori infection [12]. However, use of antibiotics in high dosages over a long period often results in drug resistance and related adverse reactions and complications, threatening the survival and quality of life of patients [13-15].

${ }^{1}$ School of Pharmacy, Inner Mongolia Medical University, Inner Mongolia China ${ }^{2}$ Affiliated People's Hospital, Inner Mongolia Medical University, Inner Mongolia China

*Correspondence: Zhenfei Wang, E-mail: ndwzf2013@126.com 


\section{Understanding $H$. pylori-associated gastritis from the perspective of traditional Chinese medicine}

In traditional Chinese medicine theory, H. pylori-associated gastritis is mainly caused by emotional injury or exogenous evil generated by improper diet. Among the exogenous evil, damp and hot are the primary pathogenic factors [16].

\section{Prevention and treatment of $H$. pylori-associated gastritis using Chinese medicine}

Table 1 presents the main works related to the treatment of $H$. pylori-associated gastritis using both traditional Chinese medicine and Western medicine.

Ye[17] studied the curative effect of Chinese medicine gargle in the treatment of $H$. pylori-associated chronic gastritis. Seventy-six patients infected with $H$. pylori were randomly divided into experimental and control groups. The experimental group was treated with traditional Chinese medicine gargle and triple therapy, while the control group was only treated with triple therapy. The results showed that the cure rate and the total effectiveness rate of the experimental group were $90 \%$ and $95 \%$, respectively. The cure rate and the total effectiveness rate of the control group were $39 \%$ and $72 \%$, respectively. The effect of traditional Chinese medicine gargle combined with triple therapy was superior to that of triple therapy. Introduction of traditional Chinese medicine gargle generates a higher cure rate and simultaneously adds a very limited cost, representing a promising new treatment method.

Zhu [18] collected 92 cases of $H$. pylori-associated gastritis patients and randomly divided them into experimental and control groups. The experimental group was treated with the prescription of invigorating spleen and clearing heat combined with probiotics, while the control group was treated with probiotics alone. It was found that the effect of combined treatment is better than that of probiotics alone, since the patients in the experimental group showed more significant improvement in clinical epigastric pain, belching,

Tab. 1: A summary of the research works on the prevention and treatment of Helicobacter pylori-associated gastritis using traditional Chinese medicine

\begin{tabular}{|c|c|c|c|}
\hline Therapeutic method & Aspects of action & Conclusion & Reference \\
\hline $\begin{array}{l}\text { Traditional Chinese medicine } \\
\text { gargle combined with triple } \\
\text { therapy }\end{array}$ & Cure rate & $\begin{array}{l}\text { The combination of traditional Chinese } \\
\text { medicine and Western medicine has } \\
\text { better curative effect }\end{array}$ & [17] \\
\hline $\begin{array}{l}\text { Invigorating spleen and clearing } \\
\text { heat combined with probiotics }\end{array}$ & Clinical symptoms & $\begin{array}{l}\text { The combination of traditional Chinese } \\
\text { medicine and Western medicine has } \\
\text { better curative effect }\end{array}$ & {$[11,18]$} \\
\hline $\begin{array}{l}\text { Si Huang Tiao Wei decoction } \\
\text { combined with proton pump } \\
\text { inhibitor triple therapy }\end{array}$ & Cure rate/eradication rate & $\begin{array}{l}\text { The combination of traditional Chinese } \\
\text { medicine and Western medicine has } \\
\text { better curative effect }\end{array}$ & {$[19,20]$} \\
\hline $\begin{array}{l}\text { Si Miao pills combined with } \\
\text { quadruple therapy }\end{array}$ & $\begin{array}{l}\text { Cure rate/eradication rate/clinical } \\
\text { symptoms }\end{array}$ & $\begin{array}{l}\text { The combination of traditional Chinese } \\
\text { medicine and Western medicine has } \\
\text { better curative effect }\end{array}$ & [21] \\
\hline $\begin{array}{l}\text { Supplemented Bupleurum } \\
\text { soothing liver powder combined } \\
\text { with } H \text {. pylori root section }\end{array}$ & Eradication rate/effectiveness rate & $\begin{array}{l}\text { The combination of traditional Chinese } \\
\text { medicine and Western medicine has } \\
\text { better curative effect }\end{array}$ & {$[22,23]$} \\
\hline $\begin{array}{l}\text { Chinese medicine } \\
\text { Jinghuaweikang capsules }\end{array}$ & Eradication rate/adverse reaction & $\begin{array}{l}\text { Chinese medicine Jinghuaweikang capsules } \\
\text { are safer }\end{array}$ & {$[24,26]$} \\
\hline $\begin{array}{l}\text { Banxia Xiexin decoction } \\
\text { combined with triple therapy }\end{array}$ & Bacteriostasis/eradication rate & $\begin{array}{l}\text { The combination of traditional Chinese } \\
\text { medicine and Western medicine has } \\
\text { better curative effect }\end{array}$ & {$[27,32]$} \\
\hline
\end{tabular}


nausea and vomiting, dry mouth, bad breath, anorexia, lassitude, diarrhea, sweating, red tongue, and so on. Additionally, experiments [11] have proved that the prescription of invigorating spleen and clearing heat can inhibit $H$. pylori in vitro, and this effect is likely due to the cold drugs from the prescription. Although data are still inadequate, the prescription of invigorating spleen and clearing heat undoubtedly has good therapeutic effect on $\mathrm{H}$. pylori-associated gastritis. The effect of invigorating spleen and clearing heat prescription and Western medicine deserves more attention.

Yang [19] designed and carried out an experiment on the treatment of $H$. pylori-associated gastritis and peptic ulcers by using Si Huang Tiao Wei decoction combined with proton pump inhibitor (PPI) triple therapy. The patients were randomly divided into three groups and treated with different methods. Then the therapeutic effects of traditional Chinese medicine and Western medicine on chronic gastritis and peptic ulcer were compared, and whether Chinese medicine can eradicate $H$. pylori was also observed. The result showed that Si Huang Tiao Wei decoction combined with PPI triple therapy can improve the cure rates of chronic gastritis and ulcers, as well as relieving the clinical symptoms of peptic ulcer. Moreover, Si Huang Tiao Wei decoction combined with PPI triple therapy displayed a significantly higher eradication rate for H. pylori than either Si Huang Tiao Wei decoction or PPI triple therapy [20]. These results indicate once again the necessity of the integration of Chinese medicine and Western medicine.

Wang [21] recruited 243 patients with $H$. pylori infection and divided them into experimental and control groups, based on treatment with either Si Miao pills combined with quadruple therapy or quadruple therapy alone, respectively. The patients in the experimental group showed significant improvement in terms of both curative effect of TCM syndrome and the eradication rate of $H$. pylori. After treatment, the eradication rate of H. pylori in the experimental group (81.82\%) was higher than that in the control group (54.84\%). In addition, the combination therapy can relieve many syndromes, such as gastrectasia, mouth odor, thirst without desire to drink, heaviness, belching, and anorexia. Thus, the combination therapy deserves further exploration for application in clinical treatment.

Shi and Liu [22] observed the efficacy of modified bupleurum soothing liver powder combined with $H$. pylori eradication in the treatment of $H$. pylori-associated chronic gastritis. A total of 120 patients with chronic gastritis were randomly divided into control group (60 cases) and experimental group (60 cases). The patients in the control group were only treated and assessed with reference to $H$. pylori eradication, while those in the experimental group were treated with the aim of assessing both $\mathrm{H}$. pylori eradication and use of traditional Chinese medicine comprising modified bupleurum soothing liver powder. The effectiveness rates of the treatment for $H$. pylori-associated chronic gastritis in the experimental and the control groups were $95 \%$ and $71.7 \%$, respectively. The result indicates that the combination treatment is superior to $\mathrm{H}$. pylori eradication treatment alone. Another group [23] compared the efficiency of PPI triple therapy, bupleurum soothing liver powder therapy, bupleurum soothing liver powder therapy and their combination therapy against $H$. pylori-associated gastritis. It was found that the combination therapy is more effective than the individual therapies in the treatment of $H$. pylori-associated chronic gastritis and peptic ulcers.

Jinghuaweikang capsule is a kind of Chinese medicine specifically developed for $H$. pylori-associated chronic gastritis. It has an excellent effect in relieving pain and invigorating the stomach [24]. Di and Guan [25] and Li [26] observed the clinical effect of Jinghuaweikang capsule on H. pylori-associated chronic gastritis in elderly patients. A total of 92 patients were randomly divided into control group and experimental group. The control group was treated with standard triple therapy, while the experimental group was treated with a combination of triple therapy and Jinghuaweikang capsule therapy. The total effectiveness rates of the control group and the experimental group were $71.7 \%$ and $91.3 \%$, respectively. In addition to enhancing the treatment effect, Jinghuaweikang capsules can also reduce adverse drug reactions and shorten treatment time.

Banxia Xiexin decoction, a famous prescription recorded in "Shanghanzabinglun" [27] and often used to treat distention and fullness, is effective in chronic gastritis therapy. Qu et al. [28] investigated the therapeutic effect of Banxia Xiexin decoction on $H$. pylori-associated gastritis. The results showed that the experimental group had a $H$. pylori infection rate of $62.7 \%$ before treatment, and the Banxia Xiexin decoction treatment generated an $80.1 \%$ elimination rate of $H$. pylori. In vitro drug sensitivity test showed that Banxia Xiexin decoction [29] had inhibitory and killing effects on $\mathrm{H}$. pylori, and the inhibitory and killing effects were 
particularly significant for Radix Scutellariae and Rhizoma Coptidis, two major components of the Banxia Xiexin decoction. Other researchers [30,31] showed that Banxia Xiexin decoction combined with standard triple therapy has a significant effect on peptic ulcer, generating a higher eradication rate of $H$. pylori and lower rate of adverse reactions than the individual therapeutic methods [32].

\section{Summary}

Both Chinese medicine and Western medicine agree that infection is caused by external pathogens [33]. During treatment, "Qing Re Jie Du" and "Fang Xiang Hua Shi" should be used first to remove exterior pathogenic factors. The difference in relation to Western medicine is that traditional Chinese medicine pays more attention to the eradication of the symptoms in terms of repairing the damaged body. The theory of traditional Chinese medicine always takes note of "evil spirits" and "righting", in addition to paying more attention to safety in the use of drugs. Treatment of $H$. pylori-associated gastritis with Chinese medicine has a more obvious mitigation effect on clinical symptoms. It has many advantages, such as high cure rate, high eradication rate of $H$. pylori, less side effects, low cost, and avoidance of drug resistance. Recently, advances in the study area show the great potential of traditional Chinese medicine in the treatment of $H$. pyloriassociated gastritis. As long as we continue to carry on the research along the footprints of the predecessors, it is sure to open up a new world of modernization of the theory of traditional Chinese medicine.

Acknowledgments: This project was supported by the Program for Young Talents of Science and Technology in Universities of Inner Mongolia Autonomous Region NJYT-17-B30.

Conflict of interest: The authors state no conflicts of interest.

Authors' contributions: Hu conducted the literature analysis, in addition to writing, discussing, and revising the manuscript of this review. Wang critically analyzed and corrected the manuscript. All authors read and approved the final manuscript.

\section{References}

[1] Potamitis G.S., Axon A.T.R., Helicobacter pylori and nonmalignant diseases, Helicobacter, 2015, 20(S1), 26-29.

[2] Roubaud B.C., Franceschi F., Salles N., Gasbarrini, A., Extragastric diseases and Helicobacter pylori, Helicobacter, 2013, 18(Suppl. 1), 44-51.

[3] Venerito M., Vasapolli R., Rokkas T., Malfertheiner P., Helicobacter pylori and gastrointestinal malignancies, Helicobacter, 2015, 20(Suppl. 1), 36-39.

[4] Chen Y.Y., The correlation between TCM syndromes of chronic non atrophic gastritis and Helicobacter pylori infection, Beijing University of Chinese Medicine, Beijing, 2013.

[5] Lin X.C., Gu Y., Li Y.H., Lin.J., Efficiency of magnifying endoscopy in diagnosis of Helicobacter pylori induced gastritis, China J. Endoscopy, 2007, 13(8), 789-791.

[6] Chen R.H., Liu M., Chen L., Shi N., Yu Q.X., Din X., Literature study on distribution characteristics of TCM syndromes and syndrome elements of Hp related chronic gastritis, China J. Tradit. Chin. Med. Pharm., 2013,28 (6), 1878-1881.

[7] Zhu J.Q., Yang J.M., Zhong J., The relationship between Helicobacter pylori phenotypes and polymorphonuclear neutrophil activity in gastric mucosa, Exp. Lab. Med., 2009, 27(1), 19-22.

[8] Chen Z.L., Efficacy evaluation and experiments about treatment for gastric disease concerning $H$. pylori with fortifying the spleen, dispelling dampness and clearing heat method, Guangzhou University of Chinese Medicine, Guangzhou, 2015.

[9] Chen J.C., The value of probiotics combined with sequential therapy in the treatment of Helicobacter pylori-associated chronic atrophic gastritis, Contemp. Med., 2017, 23(3), 97-98.

[10] Malfertheiner P., Selgrad M., Bornschein J., Helicobacter pylori: clinical management, Curr. Opin. Gastroenterol., 2012, 28(6), 608.

[11] Zhang W.D., Hu F.L., Xiao S.D., Xu Z. M., Epidemiological investigation of Helicobacter pylori infection in Chinese natural population, Mod. Dig. Intervention, 2010, 15(5), 265-270. 
[12] Georgopoulos S.D., Papastergiou V., Karatapanis S., Current options for the treatment of Helicobacter pylori, Expert Opin. Pharmacother., 2013, 14(2), 211.

[13] Zhang Y.M., Wang T.T., Ye H., Zang X. Z., Ceng H., Li J. X., et al., Jinghuaweikang capsules combined with triple therapy in the treatment of Helicobacter pylori associated gastritis: a multicenter, randomized, controlled clinical study, Chin. J. Integr. Tradit. West. Med. Dig., 2013, 21(11), 587-590.

[14] Zhang D.Y., Safety and rationality of different medication regimens in the treatment of chronic gastritis with Helicobacter pylori infection, China Contin. Med. Educ., 2017, 9(10), 178-179.

[15] Xu Y.M., Xu D.S., Jiang S.Q., Eighty-seven cases of chronic gastritis with liver qi stagnation and spleen deficiency treated by shugan Jianpi Fang, Chin. J. Exp. Tradit. Med. Formulae, 2012, 18(18), 314-315.

[16] Peng H., Deng M., Clinical study of probiotics combined with triple therapy in the treatment of Helicobacter pyloriassociated chronic gastritis in children, Proc. Clin. Med, 2016, 25(10), 795-797.

[17] Ye D.G., Observation and nursing guidance on the treatment of Helicobacter pylori infection by gargling with traditional Chinese medicine, China Health Ind., 2012, 9(07), 26-29.

[18] Zhu H.J., Effect analysis and evaluation of Chinese medicine jianpi qingre recipe combined with probiotics for the treatment of Helicobacter pylori-associated gastropathy, China Pract. Med., 2017, 12(19), 157-158.

[19] Yang H.S., Clinical study of four Huang Tiao Wei decoction combined with PPI triple therapy for the treatment of Helicobacter pylori-associated gastritis and peptic ulcer, Asia-Pacific Tradit. Med., 2017, 13(14), 124-125.

[20] Wang F., Wei B.H., Kong L.B., Zang S. W., Xiao Y. L., Effect of sihuangtiaowei decoction and PPI-combination therapy on Hp-relative gastritis and peptic ulcer disease, Chin. J. Integr. Tradit. West. Med. Gastro-spleen, 2003, 11(6), 337-339.

[21] Wang J.H., Clinical study on the treatment of Helicobacter pylori infection (spleen and stomach damp heat syndrome) with si miao pills combined with quadruple therapy, Beijing University of Chinese Medicine, Beijing, 2017.

[22] Shi P.H., Liu L., Clinical study on treatment of Helicobacter pylori infection related chronic gastritis by modified chaihu shugan powder combined with Helicobacter pylori eradication program, Chin J Exp Clin Infect Dis (Electron. Edn.), 2017, 11(5), 496-499.

[23] Wang S.D., Clinical observation of 70 cases of Helicobacter pylori infection treated by combination of traditional Chinese and western medicine, China J. Pharm. Econ., 2013, 8(01), 204-205.

[24] Jiang M.F., Efficacy of Jinghuaweikang capsules combined with triple therapy in treatment of senile chronic gastritis infected with Helicobacter pylori, Chin. J. Gerontol., 2014, 28(10), 132-133.

[25] Di Y.Q., Guan X.Q., Jinghuaweikang capsules in the treatment of senile chronic gastritis of Helicobacter pylori infection and eradication rate of HP, China Health Stand. Manage., 2017, 8(26), 108-109.

[26] Li D.X., The efficacy and HP eradication rate of Jinghuaweikang capsules senile in treatment of Helicobacter pylori infection and chronic gastritis, China Med. Pharm., 2015, 5(11), 51-53.

[27] Xing X.B., Clinical trial of typhoid fever, Tianjin science and Technology Press, Tianjin, 1984.

[28] Qu Z.W., Yu M.J., Liu C.L., Clinical study on 98 cases of Banxiaxiexin decoction for H. pylori-related positive Gastritis, J. Beihua Univ., (Nat. Sci.), 2017, 18(3), 368-370.

[29] Li L.N., Zhou H. M., Clinical study on the relationship between Banxia Xiexin decoction syndrome and HP infection, J. Tradit. Chin. Med., 1998, 39(4), 220-221.

[30] Jin H.J., Clinical observation of 40 cases of Helicobacter pylori infection-associated peptic ulcer treated with combination of traditional Chinese and western medicine, Guiding J. Tradit. Chin. Med. Pharm., 2012, 18(05), 43-44.

[31] Xiao W.Y., Wei Z., Clinical effect of Chinese and western medicine in treating chronic gastritis caused by Helicobacter pylori infection, Chin. Journal of Clinical Rational Drug Use, 2017, 10(33), 15-16.

[32] Han X.Z., Effect of combination of traditional Chinese and western medicine on Helicobacter pylori infection in patients with chronic gastritis, Henan Med. Res., 2016, 25(10), 1817.

[33] Bao M.J., Prevention and treatment of Helicobacter pylori infection by traditional Chinese medicine, Food Drug, 2017, 19(1), 71-75. 